\title{
El público de la prensa: la insoportable levedad de casi no ser
}

LA PALABRA impresa es compañera de cuna de la modernidad occidental; hermana mayor del pensamiento ilustrado; madre de la democracia. Si la imprenta facilitó la Reforma religiosa y la secularización, el periódico decimonónico consolidó la "esfera pública", lugar paradójico, público y privado, ubicuo y utópico, participativo y excluyente, en el que los ciudadanos - o más bien, algunos ciudadanos - conocieron y discutieron los avatares del poder y del dinero. El triunfo de las burguesías sobre las aristocracias y oligarquías pasó por el periódico, testigo y partícipe de las grandes transformaciones y revoluciones que desembocaron en este siglo ya moribundo, pletórico de sorpresas y mutaciones históricas, culturales, económicas, políticas, tecnológicas. La prensa prefiguró y acompañó al fordismo, la producción en línea, la sociedad de masas. El periodismo masivo ha sido compañero de viaje de la industrialización, la post-industrialización, la modernidad y la posmodernidad. La prensa - o por lo menos un sector de la misma - soñó con una parte de la humanidad en una sociedad igualitaria, solidaria y justa, sueño que al parecer se derrumbó con el muro de Berlín en 1989.

Las aspiraciones del periódico por acaparar la atención de públicos cada vez más amplios eventualmente fueron coartadas por el advenimiento de los medios electrónicos, signo y divisa del Siglo XX: La radio primero y la televisión posteriormente, fueron compartiendo y desplazando el monopolio informativo de los medios impresos, aunque no necesariamente su acceso al poder. Se ha hablado, sin embargo, de una cierta "división del trabajo" en la labor noticiosa de los medios: mientras que la radio avisa, casi simultáneamente, de la ocurrencia de un acontecimiento, la televisión muestra los sucesos; en virtud del avance tecnológico y profesional, la simultaneidad es también ya una virtud de la televisión - nos ha 
convertido en testigos presenciales de atentados a jefes de Estado, hazañas tecnológicas o deportivas, así como de guerras. Y finalmente, mediante la palabra escrita, el medio impreso sería capaz de explicar, siempre posteriormente, lo que sucedio, ampliando la información, proporcionando antecedentes y. contexto, etcétera. Desde este punto de vista, habría una gran complementariedad informativa entre medios. Lo que algunos nos preguntamos es, ¿qué proporción de la población estaría verdaderamente interesada en obtener la visión más global, explicativa y por escrito más "conceptual" de lo que cotidianamente ocurre a nivel local, nacional y mundial? Por cierto, esto no es solamente cuestión de "interés individual" en sí mismo, sino también de capacidades, competencias culturales y accesos sociales, lo que alude a una estructura de la sociedad caracterizada por la desigualdad y por lo tanto a la existencia de grandes capas sociales marginadas del "interés" por la - potencial - profundidad periodística de la prensa escrita. Lo que es un hecho ya constatado es que, prácticamente en todo cl mundo contem. poráneo, la televisión se ha acomodado como el vehículo privilegiado por los públlicos para informarse, habiéndosele otorgado una gran credibilidad, a expensas de la radio y el periódico.

Por otra parte, la misma proliferación actual de medios y de opciones tecnológicas de entretenimiento, información, educación y orientación, ha producido una múltiple segmentación de los públicos. Los llamados medios masivos, paradójicamente, cada vez amplían más su alcance potencial mediante la tecnología, pcro por su propio crecimiento explosivo tienden a cada momento a perder su masividad en la penetración y alcance reales, especializándose y aceptando de hecho múltiples divisiones del trabajo, que son también divisiones del público.

\section{La prensa en México y las élites ilustradas}

Nacido durante la Colonia, el periodismo impreso mexicano solamente comenzó a adquirir un carácter industrial y, en lo que cabe, masivo, hasta fines del siglo pasado, con el establecimiento de El Imparcial, surgido del porfirismo pero quizás anticipando su caída. De una prensa colonial de interés principalmente religioso, comercial y político-militar, pas 6 a ser después de la Independencia un periodismo principalmente político y partidista, librando con los actores históricos las grandes luchas ideológicas entre liberales y conservadores, federalistas y centralistas. El periodismo mexicano no se concentró todo en la capital de la Rcpública durante 
el Siglo XIX y principio del XX, aunque tuvo índices muy altos de centralización en las ciudades más grandes, de los estados más ricos, situación que en lo esencial no ha variado. Por otro lado, su gran interlocutor no fue - como sigue sin serlo"toda la nación", o las "masas del pueblo", sino básicamente las élites ilustradas de esas grandes ciudades, con la capital a la cabeza (Ver, por ejemplo a Ruiz Castañeda et al, 1974; Toussaint, 1989; Trejo Delarbre, 1992 y Sánchez Ruiz, 1992). La prensa ha participado en el entretejido que ha formado la "matriz de centralizaciones", finalmente estructura desigual de poder, que nos caracteriza como nación (Sánchez Ruiz, 1987). Hasta el fin de la Revolución, la prensa mexicana pudo comenzar a consolidarse como negocio, modificando su carácter político y combativo, "profesionalizándose" al tenor de la "objetividad", complementada por dosis de entretenimiento y, modernización de por medio, de amarillismo.

De la Revolución a la fecha, los medios impresos han crecido en su número y tirajes, en particular en la provincia mexicana; al paso del tiempo, se han "modernizado", adoptando las innovaciones tecnológicas del momento, que ya no nos llegan tan desfasadamente como antes, de frente a su producción e instauración en los países del capitalismo avanzado. Algo que nos preguntamos y no podemos contestarnos cabalmente es si los lectores de periódicos se han incrementado en México, proporcionalmente, con el aumento demográfico y la disminución del analfabetismo, o al mismo ritmo en que se habrían incrementado los tirajes de este medio impreso, en lo que va del siglo que ya casi termina. Esto es muy pertinente cuando, se supone, México camina hacia una "transición democrática". No podemos concebir la democracia sin un pueblo participante e informado, especialmente por un medio que es capaz de "explicar" lo que sucede, dar pormenores y múltiples puntos de vista diversos y divergentes (para los que "el tiempo no alcanza" en una nota de un minuto o dos); además de permitir o facilitar el uso de operaciones intelectuales superiores como la abstracción y la consiguiente conceptuación que, en principio, favorece la palabra escrita - de frente a la mayor concreción, "sensualidad" y momentaneidad de la imagen televisiva, por ejemplo - . Los medios electrónicos se prestan un poco más a la persuasión que a la discusión e intercambio de ideas; la prensa al contrario. En todo caso, en principio es obligación de todos los medios informativos, para propiciar la participación democrática, acoger todas las voces y posturas y hacerlas llegar a la mayor cantidad posible de ciudadanos. Lo deseable es pluralidad y pluralismo, tanto en la emisión como en la circulación 
y la recepción. Por eso, es inconcebible una sobreoferta de muchos periódicos, que son leídos por pocos receptores.

\section{El lector ausente}

Pero, ¿Cuántos, quiénes, cómo son, aquellos ciudadanos que de hecho acceden a losmensajesimpresos? ¿Qué hace la gentecon el periódico, susnoticias, supublicidad, sus secciones de entretenimiento, etcétera? ¿Influye la prensa en sus lectores? ¿En qué ámbitos puede el periódico ejercer mayor influencia? ¿Qué tan masivo es este medio de difusión? ¿Ejerce algín poder real sobre el público en general, o su "poder" proviene más bien porque las élites y los factores de poder dialogan entre sí con su mediación? ¿Cuáles son las funciones sociales que cumple el periodismo impreso entre el griceso de la población? Desafortunadamente, para el caso dc México muchas de esas preguntas no pueden contestarse en la actualidad a partir del conocimiento generado por la investigación sistemática acerca de los medios.

El público lector de los periódicos es, hasta la fecha, un gran ausente en los estudios académicos que, desde las ciencias sociales, han aboradado cl análisis de los medios masivos de difusión en México (Fuentes, 1987; Sánchez Ruiz, 1988; Aguilar Plata, 1989). La mayor parte de las investigaciones realizadas sobre la prensa sc han centrado en su historia y cvolución, su régimen jurídico, o en sus múltiples lazos con el poder y en menor medida se han ocupado en analizar su contenido (Aguilar Plata, 1989). Por ejemplo, se habla y escribe mucho acerca de diversos aspectos del contenido de los periódicos, usualmente en tono crítico, pero en realidad hay pocos estudios rigurosos que desentrañen desde la morfología hasta la ideología de la prensa. Sobre los lectores tenemos cicrtamente muy poca información sistemática, y la que existe cs más de índole descriptiva que explicativa; es decir, lo que principalmente tenemos son perfiles sociodemográficos de públicos lectores, de corte mercadotécnico. Y aún esta información, en virtud de que en su mayor parte es producida por agencias privadas, no es accesible públicamente. Más aún, el centralismo se muestra tambićn en este aspecto, pues usualmente este tipo de indagaciones descriptivas se llevan a cabo en las principales metrópolis del país, como la ciudad de México, Monterrey o Guadalajara. Desafortunadamente, por otro lado, el Censo de 1990 no incluyó preguntas referentes a los hábitos de lectura, lo que sí hizo el correspondiente al decenio anterior. El Censo Gencral de Población y Vivienda de 1980 mostró quc, del total de personas alfabctas de 15 
años y más, el 14\% no acostumbraba leer nada. De los restantes 23.6 millones de personas que dijeron leer algún tipo de material, la mayor proporción (27.3) indicó sólo cuentos, historietas, fotonovelas y revistas; $20 \%$ señalaba sólo libros y $12 \%$ sólo periódicos. Sin embargo, la proporción de personas que dijeron leer periódicos, entre otros medios impresos, alcanzó un $52.7 \%$ (12.5 millones de personas, es decir, 18\% del total de la población en 1980) (Banamex, 1986).

En los últimos años, han surgido algunas discusiones sobre la circulación de los periódicos, específicamente los del Distrito Federal, pero basadas en datos sobre tirajes, sin recurrir al posible complemento de investigaciones sobre el "consumo". Las pesquisas académicas recientes sobre recepción de los mensajes de la prensa escrita, de hecho, se pueden contar con los dedos de una mano. lo que nos queda por lo pronto, es hacer una breve referencia a algunas pocas investigaciones y debates recientes efectuados en México sobre el tema de los públicos de la prensa.

\section{Las élites ilustradas y la prensa en México, hoy}

No contamos con datos confiables sobre la circulación - una manera indirecta de estimar la lectura - de los periódicos en México. La única fuente que ha publicado con periodicidad continua los tirajes de periódicos y revistas desde el decenio de 1960, el Directorio de Medios Impresos, de Medios Publicitarios Mexicanos (MPM), es como se sabe muy inexacto, por la costumbre que ha prevalecido desde entonces, de reportar cantidades mucho mayores, en aras de la venta de espacios publicitarios. Sin embargo, ésta es ya una constatación importante, analítica y prácticamente: los periódicos, en tanto medios publicitarios que difícilmente pueden sobrevivir de la sola venta de ejemplare, necesitan en principio acreditar lectores a los anunciantes potenciales, ya sean del sector privado o del gobierno.

Desafortunadamente, durante muchos años, los investigadores - y suponemos que también publicistas y anunciantes - partimos de aquella fuente de información para tener alguna idea sobre la circulación e, indirectamente, el consumo de la prensa mexicana. Como también es sabido, no es lo mismo circulación (o distribución) que consumo o lectoría, pues por ejemplo, se dice que en el Distrito Federal cerca del 50\% de los ejemplares cotidianos de periódicos, repartidos entre voceadores y expendedores, son regresados sin venderse (Trejo Delarbre, 1990: I-III). Complementariamente, sin embargo, sabemos que cada ejemplar efectivamente vendido suele ser leído por varias personas. En 1990, la suma de los tirajes de 
diarios manifestados en el Directorio MPM era de tres millones de ejemplares. ${ }^{1} \mathrm{Sin}$ embargo, en un artículo que provocó algún escozor en el medio, Raúl Trejo (1992) calculaba que la verdadera cifra era de $751 \mathrm{mil}$. Si es verdad que casi la mitad en realidad no se venden, pero teniendo en cuenta también que usualmente un periódico es leído por más de una persona, tendríamos un número que podría estar entre 750 mil y quizas un millón de lectores de periódicos en un día común. Según el Censo de 1990 el Distrito Federal contaba ese año con cinco millones 300 mil ciudadanos mayores de 15 años con educación postprimaria. Tomando la estimación más alta, tendríamos solamente el $19 \%$ de los lectores potenciales. En el área metropolitana de Guadalajara se estima que, en promedio, circulan entre 100 y 150 mil diarios en un día típico. Una oferta estimada correspondiente al $15 \%$ de la población de 15 años y más con educación posprimaria $(947,369)$, si tomamos la cifra más alta.

De la lista de 25 diarios que utilizó Trejo para sus estimaciones, Raymundo Rivapalacio (1992: 7-14), seleccionó los nueve que él consideraba más influyentes, para los que estableció su propio cálculo del tiraje real. Los "principales diarios" capitalinos pondrían en circulación 299 mil ejemplares cada día, cifra muy cercana a los 275 mil estimados por Trejo, pero bastante lejana de los 1,155,375 manifestados a MPM por los propios nueve diarios. Estos ejercicios nos dicen que, probablemente, en promedio los periódicos mexicanos tiran cuatro veces menos ejemplares de los que suelen manifestar.

Las investigaciones sobre recepción de la prensa, en la medida en que se basan en muestras de la población, suelen producir estimaciones de lectoría relativamente sesgada, creemos nosotros, hacia los valores altos. Por ejemplo, De la Peña y Toledo (1992: 61) concluían que : “...si ponderamos a la población que declara leer periódicos por la frecuencia de lectura manifestada, que promedia 1.9 días a la semana, se tiene que en un día promedio sólo $27.5 \%$ de los informantes lee la prensa escrita". De donde inferían los investigadores que, si su muestra era representativa de la población del Valle de México, “...existen aproximadamente dos y medio millones de lectores de periódicos en un día típico". Si bien esta cifra no concuerda con el cáculo que hicimos partiendo de la estimación de tirajes de

1 En 1993, la cifra total reportada se ha reducido a 2,335,745. Ver Medios Publicitarios Mexicanos (1993). En este número de MPM no informaban sus tirajes El Heraldo ni El Sol de México en sus dos ediciones. 
Trejo (que arrojaba un $18 \%$ de los lectores potenciales, es decir, menos de la mitad de personas), podríamos concederle el beneficio de la duda, pensando en la posibilidad de que en promedio cada uno de los 751 mil ejemplares que se supone efectivamente circulan, fuese leído por tres personas. ${ }^{2} \mathrm{El}$ aspecto importante a resaltar es que, ya sean un millón o dos millones y medio (o una cifra intermedia entre éstas), el índice de lectoría de periódicos en la capital del país es enormemente bajo: entre un $18 \%$ y un $27 \%$ de la población potencialmente lectora. Téngase en cuenta que nos referimos a la entidad del país con la mayor escolaridad promedio, y con la mayor oferta de medios impresos, por lo que seguramente hay estados con índices bastante más bajos de consumo de la prensa.

En general, entonces, un primer dato que arroja la investigación disponible es un nivel bastante bajo de uso de la prensa escrita en nuestro país (Mac Kay, 1974; Rebeil Corella, 1985; RotayKorzenny, 1985; Iglesias, 1990; Toussaint, 1990; Aceves, 1990; De la Peña y Toledo, 1992). Además, debemos tener en consideración que, del total de lectores efectivos, sólo una pequeña proporción - entre una quinta y una tercera parte - leen todos o casi todos los días, mientras que una mayor parte lo hace menos frecuentemente.

\section{Mi rival es mi propia televisión}

Otra información importante que constata la investigación sobre recepción de medios en nuestro país, desde los años setenta, es la preferencia cada vez más acentuada de los públicos, por la televisión como vehículo informativo (De la Peña y Toledo, 1992: 61). La "tele" está instalada en el corazón del hogar, es parte del mobiliario y en ocasiones de la familia. La magia de la imagen otorga una ilusión de verosimilitud que no comparten los otros medios informativos. Es ya de hecho un agente de socialización fundamental en nuestras sociedades urbanas actuales. Así, por ejemplo, cuando nosotros preguntamos a una muestra de niños escolari-

2 De hecho, 3,3 personas en promedio. y aún así no es comparable nuestro $14 \%$ con el estimado $27.5 \%$ de estos investigadores, porque éste último tiene como base del álculo a la población total del Valle de México, mientras el nuestro acota solamente a los que tienen 15 años o más y tienen por lo menos educación primaria terminada, mismos que consideramos lectores potenciales de la prensa. Un problema constante que nos encontramos con la investigación por encuestas se refiere a respuestas sesgadas por "lo que se quisiera" (deseabilidad social), más que en lo que en realidad se hace. Así, hay una tendencia a decir que sí se lee, cuando en realidad no se hace, o se realiza mucho menos frecuentemente de lo que se reporta a un entrevistador. 
zados de la zona metropolitana de Guadalajara sobre a qué fuente de información le tendrían más confianza para enterarse de algo importante, la tclcvisión recibió el voto de $36.9 \%$ de los chicos, seguida por el periódico (19.7\%). Los agentes interpersonales de socialización seguían después, en los "votos de confianza" de los niños: el papá con $13.1 \%$, la mamá, $12.1 \%$, y finalmente el maestro con sólo 7.7\% del total de menciones. A una pregunta sobre credibilidad, que incluía sólo a los medios masivos como opciones de respuesta, poco menos de $60 \%$ de los infantes señaló la televisión, poco más de una quinta parte al periódico (22\%) y menos de un quinto la radio (18\%) (Sánchez Ruiż, 1989). Resultados similares han arrojado investigaciones entre sujetos de diversos rangos de edad, en varias partes del país, aunque la tendencia en los últimos años ha sido que la radio desbanque a los periódicos como segunda opción informativa.

De hecho, la década de los ochenta se puede considerar como la del "despegue" de la radio informativa en nuestro país. No es que no hubiese habido noticiarios prácticamente desde que la radio se inició en México en los años vcinte, sino que fue solamente hasta el decenio pasado que el medio dedicó muchos mayores espacios (tiempos) a la información y, en general, a los llamados "talk shows". Esto se tradujo en que la radio se "posicionó" en un lugar relevante, para cl público, como vehículo informativo. Por ejemplo, De la Pcña y Toledo (1992b: 53) encontraron que 55\% de una muestra de habitantes del Valle de México indicaron la televisión como el medio más creible; la radio ocupó cl segundo lugar con $23 \%$ y la prensa en tercero con $15 \%$ de las menciones. Casi cl mismo resultado obtuvimos nosotros en abril de 1993, con la pregunta “ ¿Cómo se informa usted sobre lo que pasa en nuestro país?", hecha a una muestra de habitantes de la zona metropolitana de Guadalajara (ver gráfica 1$){ }^{3}$ Las respuestas a una pregunta posterior, más específica, sobre cómo se informaban nuestros entrevistados acerca de los acontecimientos políticos, reforzaron el primer hallazgo (Gráfica 2).

Aún más, encontramos en la encuesta reciente cicrto indicador de "dependencia" de los públicos, con respecto a los principalcs medios de difusión. Cuando preguntamos "Si tuviera que escoger un solo medio para estar enterado de lo que

3 Tal encuesta contó con un apoyo de la Dirección de Difusión, de la Dirccción General de Comunicación Social, Presidencia de la República, para el trabajo de campo. Para cl desarrollo dcl instrumento de producción de datos colaboró el Lic. Juan Antonio Barrera, de Investigación Psico-social Aplicada. El trabajo de campo lo coordinaron Oralia Arreola y Gabricla Gómez del CEIC. 
Gráfica 1. ¿Cómo se informa usted sobre lo que le pasa en nuestro país? (Primera mención)

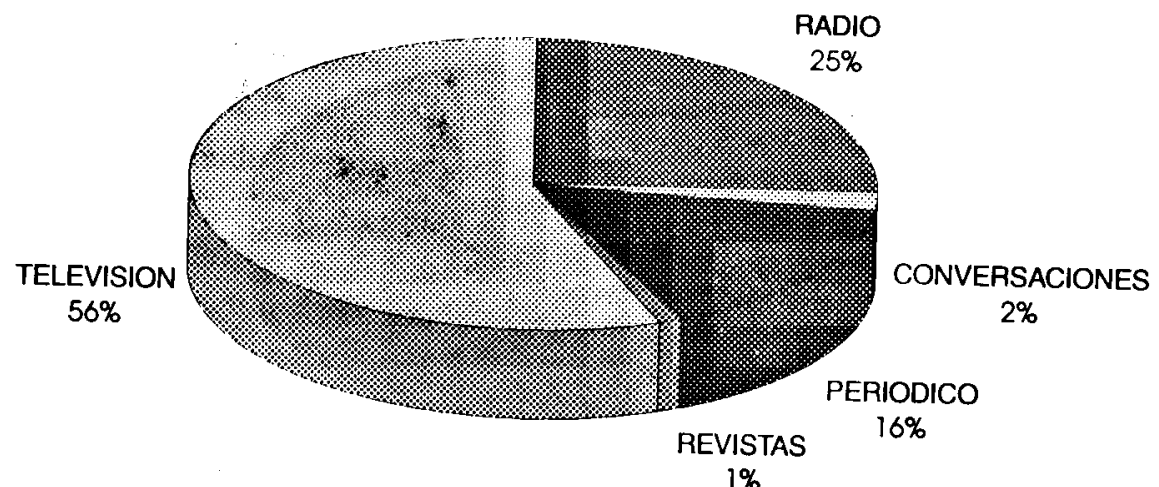

$1 \%$

$$
n=400
$$

Gráfica 2. Regularmente, ¿cómo se entera usted sobre los acontecimientos políticos que le interesan?

Sin respuesta

Periódicos

Revistas políticas

Pláticas con amigos

Pláticas en trabajo

Pláticas en escuela

Tele-noticieros Radio-noticieros

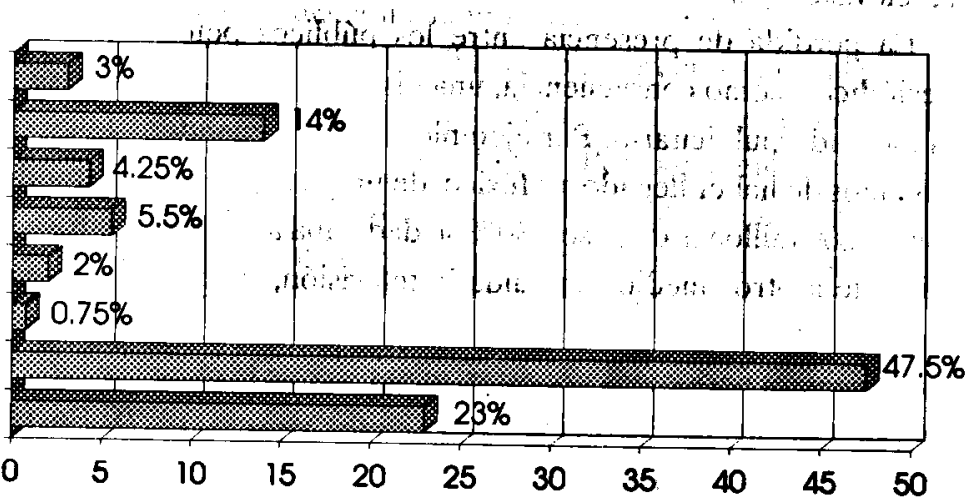

$$
n=400
$$

(Porcentajes) 
Gráfica 3. Si tuviera que escoger un solo medio para estar enterado de lo que pasa en México y en el mundo, ¿con cuál se quedaría?

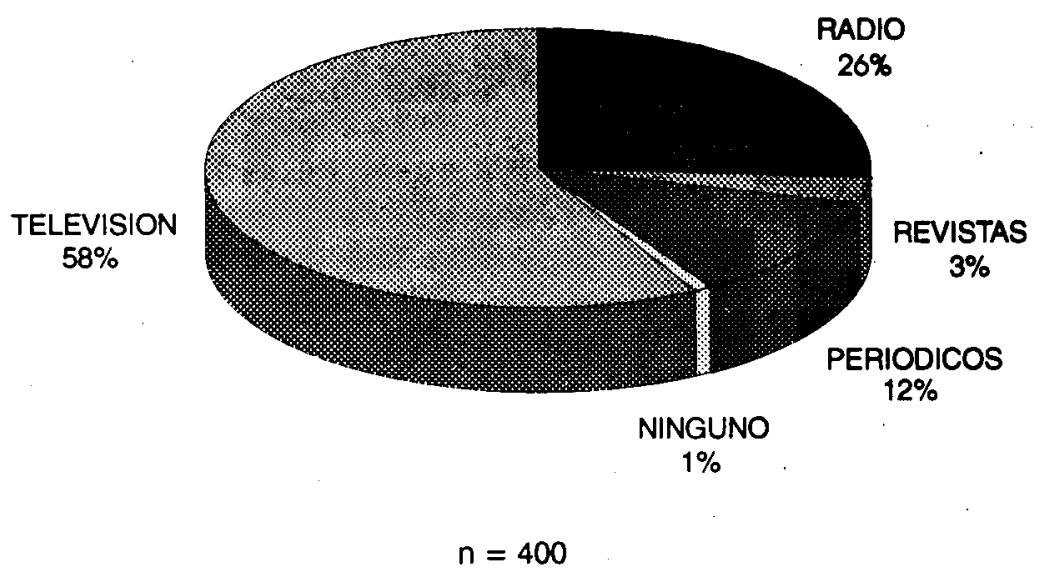

pasa en México y el mundo, icon cuál se quedaría?", sólo el $12 \%$ señalo los periódicos, el $26 \%$ indicó la radio y cerca del $60 \%$ la televisión (ver Gráfica 3). En segunda opción, los periódicos subieron a una quinta parte pero siguieron siendo el tercer lugar, manifestándose una mayor dependencia por la radio (43\%) y por la televisión (30\%).

La pérdida de presencia entre los públicos potenciales ha traído para los periódicos, como consecuencia, una disminución continua de su participación en el mercado publicitario. Por ejemplo, en 1952, cuando la televisión tenía apenas dos años de haber llegado a México, de un gasto publicitario total de aproximadamente 800 millones de pesos, $30 \%$ se dedicaba a la prensa, $40 \%$ a la radio y el $30 \%$ restante a otros medios, incluida la televisión, según datos proporcionados por Mejía Barquera (1981). Para 1963, de una facturación de 1,600 millones de pesos, $34 \%$ fue para periódicos y revistas, mientras que la radio recibi6 $23.8 \%$ y la Tv sólo un poco menos (22.3\%) (Enciclopedia de México, 1977). En 1972, de un gasto total dedicado a medios de 3,075 millones de pesos, los medios impresos recibieron $31 \%$, la radio bajó a un $19 \%$ y la televisión comenzó a mostrar su dominio del gasto 
publicitario con $39 \%$ del total. ${ }^{4}$ A principios de los ochenta ya estaba situada la televisión en el nivel que ha mantenido prácticamente hasta la fecha, con más del $60 \%$ del gasto publicitario (65\% en 1981), mientras que la radio obtenía un $15 \%$ y los impresos solamente un 12\% (Florida, 1981:11). En la actualidad los periódicos, al parecer, reciben alrededor del $13 \%$ del gasto total de publicidad vehiculado por las agencias, de 4.4 mil millones de viejos pesos aproximadamente; de esta cantidad, $63 \%$ corresponde a la televisión y un $13 \%$ a la radio. ${ }^{5}$ Esto se refiere solamente a la publilcidad comercial, particularmente aquella que se contrata por medio de las agencias publicitarias. Esta aclaración es pertinente, porque por otra parte, quizas una cantidad igual o mayor a los 576 millones de viejos pesos que representa ese $13 \%$, signifiquen para los periódicos los ingresos por la llamada "publicidad política" - de hecho propaganda - que incluye principalmente desplegados y gacetillas o inserciones pagadas.

Al menos algunos periódicos parecen poder prescindir tanto del público lector como de la publicidad comercial, y ser rentables. Dice Rivapalacio (1992: 8):

Por raro que suene aunque su tiraje sea reducido, la prensa como empresa es un gran negocio. Veamos, por ejemplo, el caso de un periódico de la ciudad de México, con un tiraje cotidiano de 5,000 ejemplares. Confidencialmente, el periódico informó que su ganancia fue casi un millón de dólares en el primer trimestre de 1989. ¿Cómoes posible?

Desde luego, cl diario al que se refiere la cita es uno de los varios que se consideran "nacionales" y que reportan una circulación cuando menos cuatro veces superior a la real. Niveles de ingresos como aquel son posibles debido precisamente al gasto gubernamental, que opera en última instancia irracionalmente, subsidiando con frecuencia periódicos con muy poca o nula circulación. Personalmente creemos que, en la medida en que el Estado mexicano y los diversos niveles de gobierno, racionalicen más su gasto, posiblemente no pase mucho tiempo sin que los medios impresos comiencen a preocuparse más por sus lectores y su circulación y consumo efectivos. Por lo pronto, hemos visto que la reducción de sus lectores ha llevado a una consecuente disminución de la proporción del gasto publicitario comercial en los periódicos. Por otra parte, los diversos sectores activos en política, especial-

4 World Advertising Expeditures (1974). Notar que la proporción de impresos incluye periódicos y revistas, aunque la mayor parte, suponemos, era para los primeros.

5 De hecho, los datos son de 1989 o 90 . Ver El Financiero del 6 de febrero de 1991. 
mente el gubernamental y los partidos políticos, a partir de 1988 poco a poco han ido tomando más en cuenta la opinión pública amplia, es decir, de grandes capas de la población, para normar algunas de sus acciones y decisiones, en lugar de la "opinión pública" de solamente ciertos líderes (políticos, empresariales, etcétera) o de relativamente pequeñas élites. En este sentido, podría ser el caso de que pronto la "publicidad política" - o por los menos una cierta porción de ella - se asigne solamente a aquellos medios que, efectivamente, lleguen a amplias capas de la población. Esto podría traer una competencia más real, al margen del “proteccionismo político" (Gutiérrez, 1993), que a su vez podría sacar del mercado aquellos que no tuvieran capacidad de llegar, efectivamente, por lo menos a ciertos segmentos de la población que interesaran a los anunciantes. Cuando estamos inmersos en un proceso de integración desigual, en un mercado norteamericano mediante el Tratado de Libre Comercio, todas las ramas industriales, comerciales yde servicios, incluidos los medios, tendrán que adquirir experiencia y capacidades competitivas y ya no será suficiente el inflar tirajes o hacer llegar ejemplares a las oficinas de prensa, para que el periodismo escrito, además de periodismo - cuando efectivamente lo sea - , pueda continuar siendo un negocio rentable.

\section{¿Calidad, no cantidad?}

Por otra parte, como hemos mencionado desde el principio, si bien el público del periodismo impreso no es numéricamente abundante, sí tiene una relativa importancia cualitativa. En líneas generales, las investigaciones disponibles muestran que los lectores de periódicos suelen ser en promedio personas más educadas y de ingresos relativamente altos, aunque por ejemplo el tipo de periódico puede hacer una diferencia. Asimismo, a mayor escolaridad, es mayor el consumo de la información de la prensa (en términos de días a la semana y en minutos al día) (De la Peña y Toledo, 1992b; Sánchez Ruiz, 1993).

Hay mayor propensión entre hombres que entre mujeres a leer los periódicos, aunque en los entornos urbanos tiende a disminuir la brecha. Entre las mujeres, es un poco mayor la tendencia a informarse por medio de la televisión y de la radio, que de la prensa. Se sabe en general que las élites decisoras, tanto en el sector privado como en el gobierno, son lectoras más o menos constantes de la prensa.

Sin embargo, habría que aclarar que, en investigaciones que comparan el consumo de diversos medios, a pesar de que se suele encontrar que los estratos 
más altos de educación e ingresos acostumbran más que los estratos más bajos la lectura del periódico, también entre aquéllos se encuentra que son la televisión y la radio los vehículos preferidos para informarse (ver Gráfica 4). En general, en una

Gráfica 4. ¿Cómo se entera usted sobre los acontecimientos políticos...?

Por estrato económico

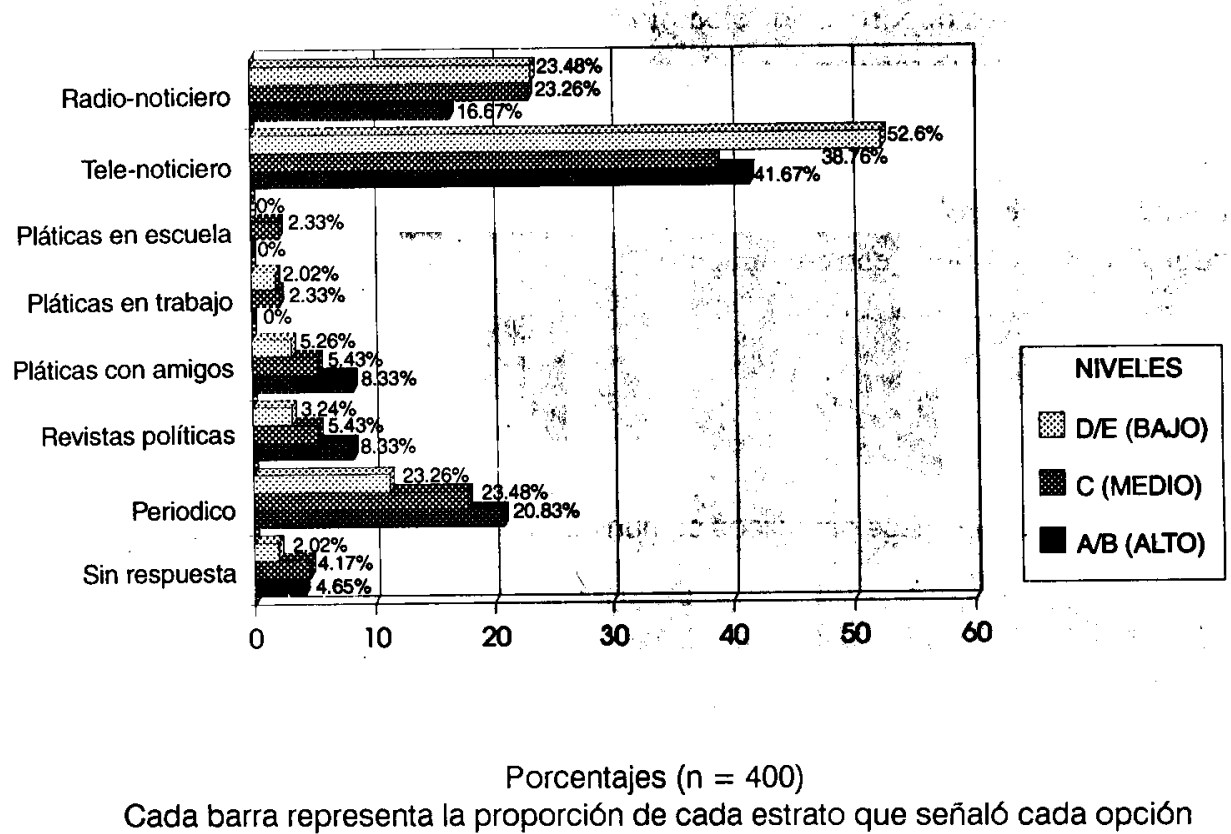

encuesta reciente que realizamos en el área metropolitana de Guadalajara, la televisión apareció como la que más cumple una serie de funciones para los entrevistados: información, aprendizaje, orientación y entretenimiento. No obstante, entre la submuestra de lectores de periódicos, éstos parecen cumplir satisfactoriamente tales funciones. A pesar de que una relativamente alta proporción de los respondientes manifestaron dudas sobre si los periódicos " comunican la verdad de los hechos que suceden" (31\% no, 48\% "tal vez", $21 \%$ sí), casi la mitad de las personas están de acuerdo en que este medio "ayuda a comprender lo que 
pasa en el país". Una porción igual piensa que "los periódicos ofrecen contenidos educativos", y $68 \%$ de ellos están de acuerdo en que "enseñan cosas interesantes". Asimismo, la mayoría de los entrevistados reportaron que en el periódico encontraban entretenimiento y que su lectura hace que "el tiempo pase rápidamente" (Gráficas 5, 6, 7, 8, 9 y 10). De hecho, este dato nos lleva a la constatación de que el periódico no es solamente un vehículo informativo "serio" para sus lectores, sino que también esperan de su lectura cierta dosis de entretenimiento. Esto se corrobora con el hecho de que, cuando uno pregunta a las personas cuál sección prefieren más, los deportes, por ejemplo, suelen aparecer en primero o segundo lugares. a la par que secciones más "serias", como la de internacionales. A fin de cuentas, sin necesidad de banalizar demasiado los contenidos del medio impreso, estas expectativas del público lector invitarían al mantenimiento de un cierto equilibrio entre secciones y tipos de contenido, especialmente entre lo informativo "duro" y lo que brinda entretenimiento.

Gráfica 5. "Los periódicos comunican la verdad de los hechos"

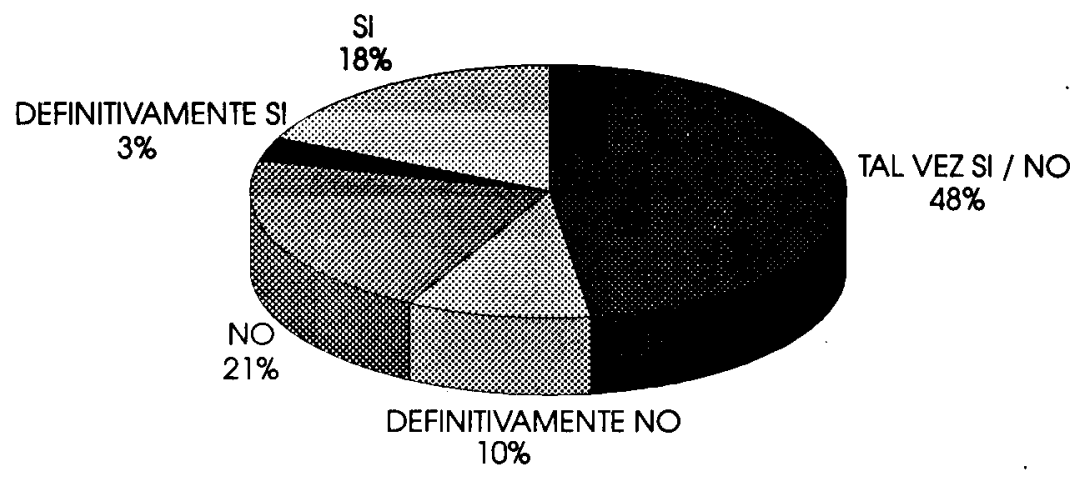


Gráfica 6. "Los periódicos ayudan a comprender las cosas que pasan en el país"

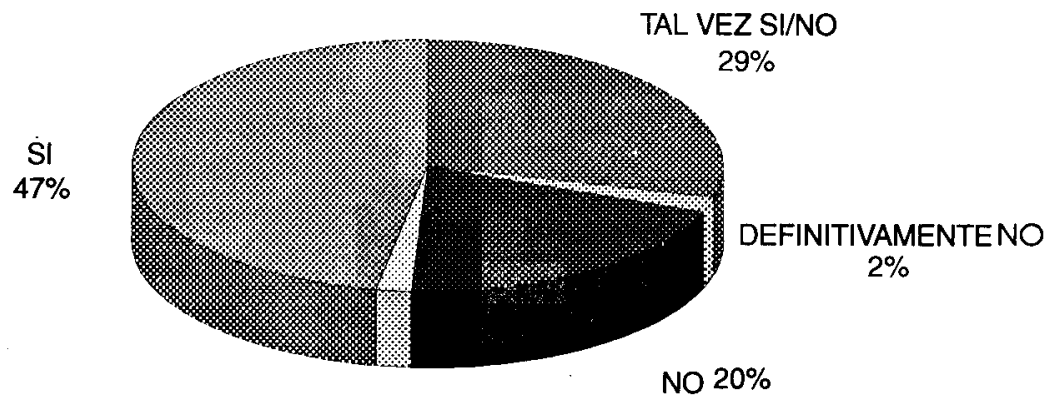

DEFINITIVAMENTE SI

$2 \%$

$$
n=185
$$

Gráfica 7. "Los perí́dicos ofrecen contenidos educativos"

DEFINITIVAMENTE $\mathrm{SI}$ $5 \%$

DEFINITIVAMENTE NO

$3 \%$

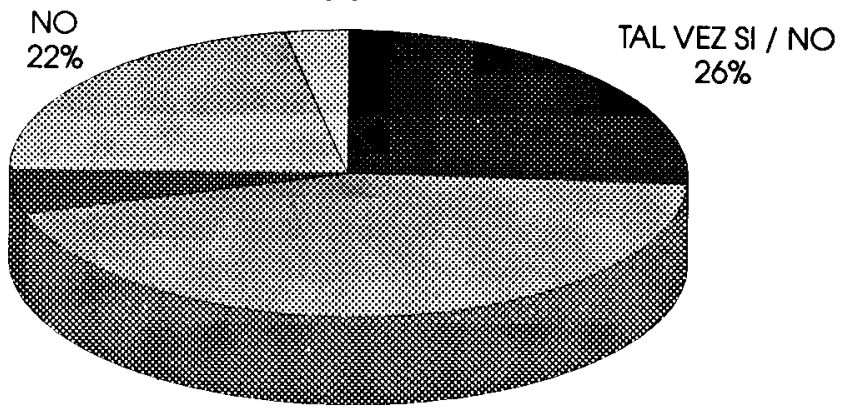

$\mathrm{SI}$

$44 \%$

$$
n=185
$$


180 El público de la prensa: la insoportable levedad de casi no ser

Gráfica 8. "Los periódicos enseñan cosas interesantes"

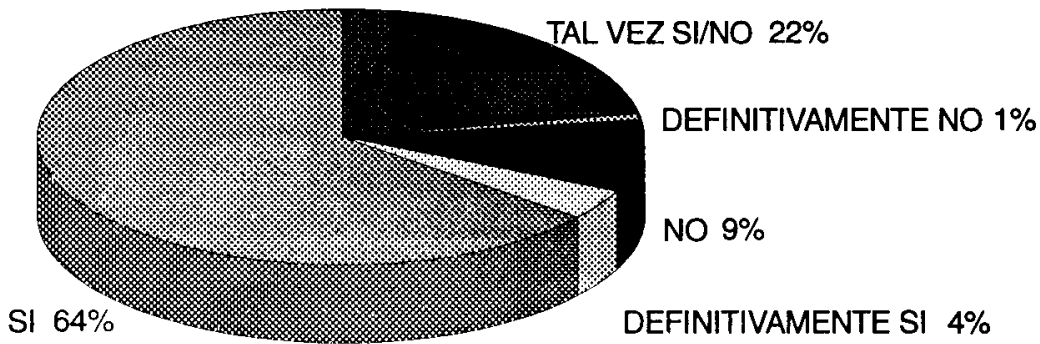

$$
n=185
$$

Gráfica 9. "Los periódicos brindan entretenimiento con los temas que ofrecen"

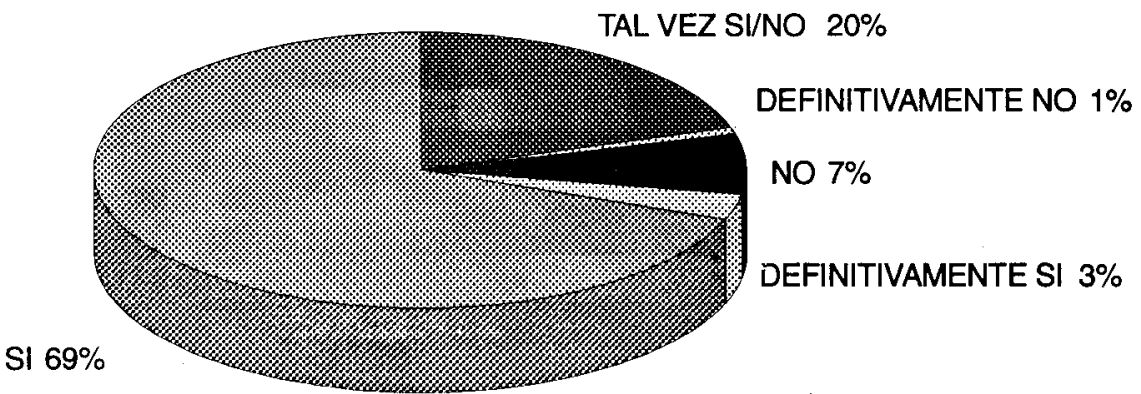

$$
n=185
$$


Gráfica 10. "Los periódicos hacen que el tiempo pase rápidamente"

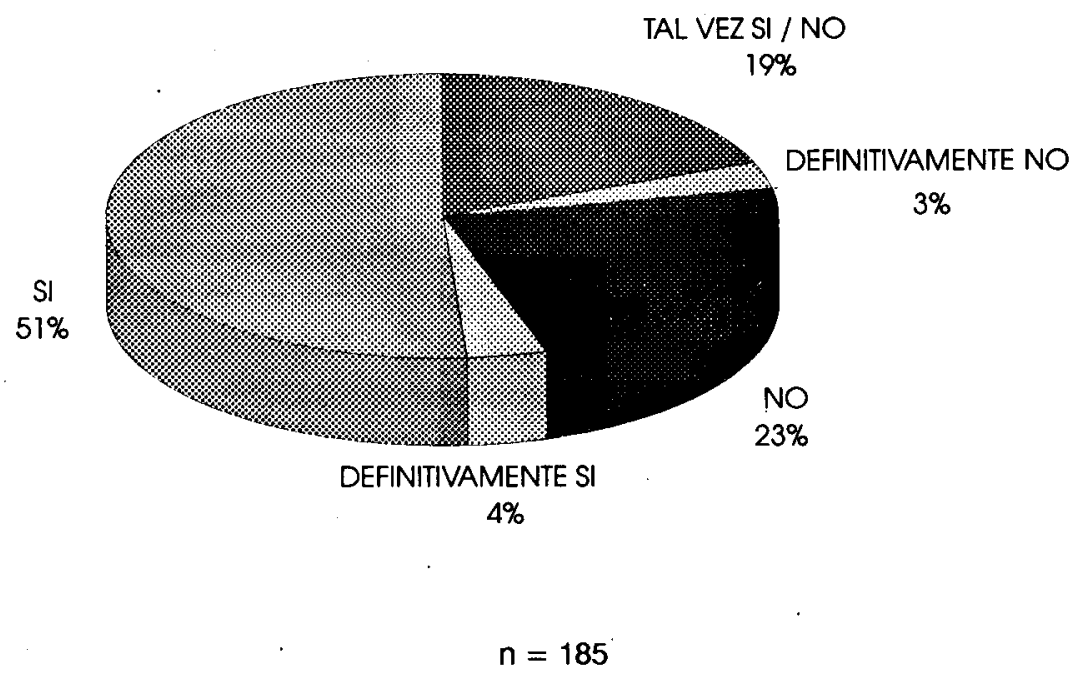

\section{A manera de conclusión}

Hemos descrito algunos aspectos relevantes sobre el público de la prensa, que se desprenden de la investigación académica accesible. Corroboramos que, en general, los lectores son desconocidos, quizá en proporción a su misma escasez, en un país que se precia de estar a punto de vencer el analfabetismo. Esta situación llama a dos exhortaciones. A los investigadores académicos, que dentro de la ciencias sociales analizan las formas de existencia y operación social de los medios de difusión, a no olvidar este medio que tanto tiene todavía por contribuir, especialmente al proceso democratizador en nuestro país. Por otra parte, en la medida en que estamos convencidos de que el proceso de globalización que envuelve aceleradamente a nuestro país, implica el desarrollo de capacidades competitivas que en decenios anteriores no eran precisas para sobrevivir en los mercados, es necesario que los directores y decisores de nuestros periódicos dejen de confiarse en los recursos tradicionales para asegurar la rentabilidad financiera. Ya sean vistos 
en términos de "mercado", o - mejor aún - como receptores potenciales de un proceso de mayor participación democrática en la vida nacional, los lectores deben recibir una mayor atención de parte de nuestros periódicos y periodistas.

Pero la falta general de hábitos de lectura de que adolece nuestro pueblo no es de hecho resultado de las acciones u omisiones de los empresarios del periodismo, sino de un sistema educativo que no los genera como un aspecto nodal para crear ciudadanos bien informados y activos en todas las esferas de la vida social. Hay una asignatura pendiente del sistema educativo mexicano en este aspecto, que en el momento en que seaatendido, suponemostendrá eventualmente como resultante el ascenso de la lectoría de periódicos. Un pueblo que no lee es finalmente analfabeta funcional, aunque sus estadísticas oficiales muestren lo contrario. Un pueblo con periódicos, pero sin lectores, está condenado irremediablemente al yugo del autoritarismo y al atraso material.

\section{Referencias bibliográficas}

ACEVES Francisco (1990): "Los tapatíos y la prensa". Guadalajara: CEIC - U. de G. Informe de investigación.

Aguilar Plata, Blanca (1989): "Subsistema prensa: Descripción y clasificación del conocimiento existente sobre la prensa en México". Informe parcial del Estudio Comparativo de los Sistemas de Comunicación de Brasil y México. México: INTERCOM/CONEICC.

BANAMEX (1986): México Social, 1985-1986. Indicadores Seleccionados. pp.227279. México: Banamex.

DE la PEÑa Ricardo y Rosario Toledo LaguaRdia (1992b) “Hábitos de lectura de periódicos en el Valle de México”. Intermedios Núm. 3, p.53 (agosto).

yRosario TOLEDOLAGUARDIA(1992a): "Hábitos de lectura de periódicos en el Valle de México". Intermedios Núm. 4, p. 61 (octubre). 
ENCIClOPEDIA DE MÉXICo (1977): "La publicidad". Enciclopedia de México, Tomo X, pp. 448.66 .

FLORIDA John, A. (1981): "Advertising and the mass media". En John H. CHRISTMAN (ed.). Business México. México: American Chamber of México.

FUENTES Raúl (1987): La investigación de comunicación en México. Sistematización documental, 1956-1986. México: Ediciones de Comunicación.

GuTIÉRREZ ESPÍNDOLA José Luis (1993): “La Prensa y sus lectores. Una reflexión desde la perspectiva de la opinión pública y la democracia", Conferencia presentada en el II Simposio Internacional de Editores de Periódicos Diarios, México, D.F., 3-5 Junio.

IgLESIAS Norma (1990): Medios de comunicación en la Frontera Norte. México: Fundación Manuel Buendía / Programa Cultural de las Fronteras.

MACKAY Lamar (1974): "Uso de los medios de comunicación colectiva en Guadalajara". Universidad Autónoma de Gadalajara, Informe de Investigación.

MALKIN Elizabeth (1992) "Media costs. Advertisers pay a numbers game full of mystery and surprise". Business Mexico Vol. II, Núm. 9, p. 11 (septiembre).

Medios Publicitarios MeXicanos (1993): Tarifas y datos, medios impresos. Núm. 1. México: Medios Publicitarios Mexicanos (febrero).

MeJf́a BARquera Fernando (1981): La industria de la radio y la televisión y la política del Estado mexicano (orígenes y desarrollo). Tesis de Licenciatura, UNAM, FCPS.

REBEIL CoRella (1985): "Los medios de comunicación en la vida de la sociedad receptora". México: Universidad Autónoma Metropolitana, Xochimilco, informe de investigación. 
RIVAPAlACio Raymundo (1992): "La prensa mexicana, icontrolada?". Revista Mexicana de Comunicación Año 5, Núm. 25, pp. 7-14 (septiembre-octubre).

RoTA Josep y Felipe KoRZENNY (1985): “Media use by adults in Mexico City as a function of socio- economic status and sex", Ponencia presentada en la XXXV Conferencia anual de la International Communication Association, Honolulu, Hawaii.

Ruiz Castañeda et al (1974): El periodismo en México. 450 años de historia. México: Editorial Tradición.

SÁNCHEZ RuIZ Enrique E.(1987): Centralización, podery comunicación en México. Guadalajara: Universidad de Guadalajara, Cuadernos del CEIC, Núm. 3.

- (comp.) (1988): La Investigación de la Comunicación en México: Logros, Retos y Perspectivas. México: Ediciones de Comunicación / Universidad de Guadalajara.

(1989): "Televisión y representaciones políticas de escolares tapatíos". Comunicación y Sociedad Núm. 7.

(1992): La Prensa Diaria de Guadalajara: Desarrollo y Perspectivas. Guadalajara: El Colegio de Jalisco, Cuadernos de Estudios Jaliscienses, Núm. 12.

(1993) "Hábitos de Consumo de periódicos de habitantes de la Zona Metropolitana de Guadalajara". Guadalajara: CEIC, Universidad de Guadalajara. Primer Informe de la Investigación: "Usos y Funciones del Periódico". Inédito.

TousSAINT Florence (1989): Escenario de la Prensa en el Porfiriato_México: Fundación Manuel Buendía / Universidad de Colima.

- (1990): Recuento de Medios Fronterizos. México: Fundación Manuel Buendía / Programa Cultural de las Fronteras. 
TREJo Delarbre Raúl (1990): "Periódicos: ¿Quién tira la primera cifra?". Cuadernos de Nexos Núm. 24, pp. I-III (junio). (1992): “La expresión pública”. Intermedios Núm. 3 (agosto).

WORLD ADVERTISING EXPEDITURES (1974): World Advertising Expenditures. Starch-INRA Hooper-IAA. 